\title{
Age-period-cohort analysis of kidney cancer deaths attributable to high body-mass index in China and U.S. adults
}

Xiaoxue Liu', Yong Yư ${ }^{2}$, Minsheng Wang ${ }^{3}$, Fang Wang ${ }^{1}$, Sumaira Mubarik${ }^{1}$, Yafeng Wang ${ }^{1}$, Runtang Meng ${ }^{1}$ and Chuanhua $Y u^{1,4^{*}}$

\begin{abstract}
Background: Statistical data on burden of kidney cancer and the relavant risk factors are valuable for policymaking. This study aims to estimate kidney cancer deaths and high body-mass index (BMI) attributable to the deaths by gender and age group in China adults, compared with U.S.

Methods: We extracted kidney cancer data (1990-2017) about the age-standardized rates using the comparative risk assessment framework of the 2017 Global Burden of Disease study. We performed an age-period-cohort (APC) analysis to estimate trends of kidney cancer mortality attributable to high BMI.

Results: During 1990-2017, age-standardized mortality rate of kidney cancer was increasing in China but decreasing in U.S. The mortality attributable to high BMI in China showed a general increasing trend, while that in U.S. men was increasing and tended to be stable in women since 1995. APC analysis showed a similar pattern of age effect between China and U.S. adults, which substantially increased from 20 to 24 to 90-94 age group. Differently, the period effect rapidly increased in China than U.S. adults during 1990-2017. The cohort effect peaked in the earlier cohort born in 1902-1906 in China, and it declined consistently in U.S. with exception of 1902-1906 and 1907-1911 birth cohort.

Conclusions: The kidney cancer deaths attributable to high BMI, and period effect have been generally increasing in China adults, compared with U.S. adults in which the trend tends to be stable in recent years. The rapid aging may also intensify the increasing trend of kidney cancer death in China. Effective measures should be conducted on body weight control and care for kidney cancer prevention.
\end{abstract}

Keywords: Kidney cancer, Mortality, High BMI, Age-period-cohort effect, Trend

\section{Background}

Kidney cancer has been the common cancers in the world $[1,2]$. There are 48,210 new cases and 17,168 deaths in China in 2017, accounting for $12.27 \%$ and $12.39 \%$ of 393 , 042 new cases and 138,528 deaths worldwide, respectively

\footnotetext{
* Correspondence: yuchua@whu.edu.cn

'Department of Epidemiology and Biostatistics, School of Health Sciences,

Wuhan University, No. 115, Donghu Road, Wuhan 430071, China

${ }^{4}$ Global Health Institute, Wuhan University, Wuhan 430072, China

Full list of author information is available at the end of the article
}

[2]. Kidney cancer mainly includes renal cell carcinoma with a proportion of $90-95 \%$ [3]. The incidence of kidney cancer has increased year by year in recent years, rising by $2 \%$ to $3 \%$ compared with 10 years ago [4]. The mortality of kidney cancer has been stable globally since the 1990s, while it decreases in most countries in recent years [5]. In China, both incidence and mortality of kidney cancer seemed low, but previous study reported incidence of kidney cancer had greatly increased [6]. The mortality trend of kidney cancer remains unknown. 
Established risk factors for kidney cancer mainly include tobacco smoking, obesity/overweight, hypertension and chronic kidney disease [5, 7-10]. It is currently accepted that there is a global epidemic of obesity [11]. The prevalence of overweight or obesity in China adults is also rising greatly during 1993-2014 [12, 13]. It is being concerned that obesity increased the risk of kidney cancer $[8,14]$. This relationship is attributed to abnormal secretion of adipokines, insulin resistance, higher estrogen level among overweight/obesity individuals [9, 10]. However, with a rapid urbanization and transition to western dietary and lifestyle in China, people experienced an increasing exposure to obesity [6, 15], and trends of kidney cancer death remains unknown. The prevalence of obesity/overweight is different between China and U.S. populations. Therefore, we aim to further estimate the time patterns of kidney cancer mortality attributable to high BMI to present the relative risk due to age, period and cohort of the mortality in China, compared with U.S., which could provide epidemiological evidence for kidney cancer prevention and control.

\section{Methods}

\section{Data sources}

This study obtained kidney cancer data from global burden of disease (GBD) 2017 study. GBD 2017 study provided a comprehensive estimation of annual incidence, prevalence, mortality for causes of death and the corresponding risk factors in 195 countries and territories during 1990-2017 [16, 17]. The GBD study summaries burden of disease for global populations among different causes, locations, ages, and sexes [18]. The authors declare all the data used in the study was deidentified. Ethics was not required because the data was publically available.

The age-standardized mortality rates (ASMR) of kidney cancer attributable to high BMI were obtained from GBD 2017 [19]. The GBD study attributes each death to a single underlying cause that began the series of events leading to death, in accordance with ICD principles. Kidney cancer was defined as the International Classification of Diseases of the 10th revision (ICD-10) code C64 and code 189 in ICD-9 [16]. The original mortality database is composed of vital registration (VR), verbal autopsy (VA), registry, survey, police, and surveillance data $[1,20]$. The GBD study organizes causes of death in a hierarchical list (four levels), which was further refined to separately estimate causes with substantial policy interest or high levels of burden. To predict the level for each cause of death, GBD 2017 used the Cause of Death Ensemble model (CODEm) to systematically test a large number of functional forms and permutations of covariates [16]. The original data of kidney cancer mortality in
China population was mainly from the Cause of Death Reporting System of the Chinese Center for Disease Control and Prevention (CDC), Disease Surveillance Points (DSPs) and the Maternal and Child Surveillance System, which are considered to be nationally representative [21]. Mortality data sources of both China and U.S were classified into cancer groups according to the International Classification of Diseases and estimated by GBD study. To increase the comparability of the two regions, age-standardized mortality rates were calculated adjusted to the GBD 2017 global standard population using the direct method. In this analysis, mortality data of the population aged under 20 years old was excluded for both China and U.S. because kidney cancer diagnosed in children is rare. The mortality of people aged above 95 years old was also excluded because these data couldn't meet APC analysis.

\section{Attributable burden}

The GBD study incorporated the comparative risk assessment framework previously to quantify the burden of several causes and impairments attributable to 84 environmental, occupational, metabolic, and behavioral risk factors. Systematic literature search was performed in PubMed to find the evidence for kidney cancer deaths due to the attributable risk factors. For each included study, the proportions of kidney cancer cases induced by the specific risk factors were calculated with exception of outliers or data that did not meet the inclusion criteria [2]. Briefly, after assessing the casual evidence in each risk-outcome pair, we analyzed the attributable burden of kidney cancer deaths to high BMI. Overweight and obesity are defined by measures of weight and height that provide an index of one's mass, referred to as a BMI. In this study, based on comparative risk assessment from GBD 2017 study, high body-mass index (BMI) for adults (ages 20+) is defined as BMI greater than theoretical minimum risk exposure level: $20-25 \mathrm{~kg} / \mathrm{m}^{2}$ [22].

\section{Statistical analysis}

In the descriptive analysis, the age-standardized mortality rate (per 100,000 population) was calculated according to the direct method by summing up the products of agespecific rates ( $\alpha_{i}$, where $i$ denotes the $i$ th age class) and the number of persons $\left(\beta_{i}\right)$ in the same age subgroup $i$ of the chosen reference standard population, followed by dividing the sum of the standard population weights, i.e., [23].

$$
A S M R=\frac{\sum_{i=1}^{A} \alpha_{i} \beta_{i}}{\sum_{i=1}^{A} \beta_{i}} \times 100,000
$$

Standardization was considered imperative for this study as it eliminates the bias when comparing the rates between the two areas. 
The APC model could be expressed as:

$$
Y_{J}=\mu+a \text { age }_{j}+\text { Bperiod }_{j}+\text { rcohort }_{j}+\varepsilon_{i}
$$

where $Y_{J}$ denoted the response variable-the net effect on colorectal cancer mortality for group $j, a, \beta$ and $\gamma$ denoted the coefficient of age, period and cohort of APC model, respectively, and $\mu$ denoted the intercept of the model. $\varepsilon_{i}$ denoted the residual of the APC model.

In this model, the cohort can be expressed by age and period; that is, cohort = period-age. An on-identification problem may still exist as there is a linear relationship between the age, period and cohort. In our study, APC model with an intrinsic estimator (IE) method was used to solve the collinearity problem, which is based on estimable functions and the singular value decomposition of matrices [24].

In this analysis, age reflects variations in vital rates, based on that mortality risk increases with the process of ageing. Period effects represent influential factors, including complex sets of historical events and environmental factors, that simultaneously affect all age groups. Cohort effects represent variations across groups of individuals born in the same year or years. The age, period and cohort effects influence morbidity and mortality risks of disease in specific ways, especially period effect represent complex sets of historical events and environmental factors [1]. APC analysis was used to decompose the three trends in mortality and provides unbiased and relatively efficient estimation results [25]. The conventional approaches include two-factor models (age-period (AP), age-cohort (AC), and periodcohort (PC) models) and constrained generalized linear models (CGLIMs). APC model was commonly selected for estimating age, period and cohort effect of disease data [24, 26]. Goodness-of-fit statistics and the best-fitting model was presented in Supplementary Table 1.

In this work, age-specific rates of kidney cancer mortality attributable to high BMI were classified by consecutive age groups $(20-24,25-29, \ldots, 90-94), 5$-year periods (1992, 1997, 2002, 2007, 2012, 2017), and correspondingly 5-year birth cohort groups (1902-1906, 1907-1911, ..., 1997-2001). The estimated coefficients for the age, period and cohort effects by APC analysis was shown in Supplementary Table 2A and 2B, and then these coefficients were calculated to the exponential value $\left(\exp (\right.$ coef. $\left.)=\mathrm{e}^{\text {coef. }}\right)$ which denotes the mortality relative risk (RR) of a particular age, period, or birth cohort relative to each average level (see Tables 1 and 2). For example, for period effect of China men, the risk of kidney cancer due to high BMI in 2017 was 6.23 (exp. $\left(\beta_{2017}-\right.$ $\left.\left.\beta_{\text {mean }}\right)-\left(\beta_{1992}-\beta_{\text {mean }}\right)=\exp .\left(\beta_{2017}-\beta_{1992}\right) \approx 6.23\right)$ times the risk in 1992 . This analysis was performed by Stata 14.0 software (StataCorp, College Station, TX, USA).

\section{Results}

Age-standardized mortality rates of kidney cancer

Figure 1 shows ASMR of kidney cancer in China and U.S. for both sexes at all ages during 1990-2017. According to GBD 2017, the ASMR of kidney cancer increased from $0.64 / 100,000$ in 1990 to $0.94 / 100,000$ in 2017 in China, and it decreased from 3.21/100,000 in 1990 to $3.12 / 100,000$ in 2017 in the U.S. In 2017, the ASMRs of kidney cancer in U.S. (4.55/100,000 in men; $1.93 / 100,000$ in women) were much higher than China $(1.29 / 100,000$ in men; $0.62 / 100,000$ in women) for both sexes. Overall, the ASMR of kidney cancer was increasing in China for both sexes, while the ASMR in the U.S. decreased and tended to be stable during the same period. Thus, the prevalence of risk factors for kidney cancer may still impact the mortality in Chinese people.

\section{Trends in mortality of kidney cancer attributable to high BMI}

Trends in ASMR of kidney cancer attributable to high BMI from 1990 to 2017 for China and the U.S. are shown in Fig. 2. The highest rate in China was $0.12 / 100$, 000 in men and $0.09 / 100,000$ in women in 2017. In the U.S., the highest rate was $1.18 / 100,000$ in men and 0.70 / 100,000 in women during 2001-2005. China showed a low attributable mortality, compared with the U.S. In overall, a general increasing trend was observed, except for U.S. women, although it shows a low level of agestandardized mortality rate of kidney cancer attributable to high BMI in China compared with the U.S.

\section{The results from APC analysis of kidney cancer mortality attributable to high BMI}

Age, period (year of death), and cohort (year of birth) are three independent factors that have been found to be associated with cancer mortality, and, therefore, each of these factors may affect trends in cancer mortality. We mentioned before that as we used APC model with the IE method to eliminate the non-identification problem as there is a linear relationship between the age, period and cohort. The three independent effects were presented:

\section{Age effect}

Age effect on the mortality showed an increasing trend in both China and U.S. adults (Fig. 3a, Tables 1 and 2). The age effect indicates the RR of mortality attributable to high BMI (high-BMI-attributable mortality) varies from younger and older age groups. Generally, a similar pattern of age effect was observed between China and U.S., as well as genders. From 20 to 24 to $90-94$ age group, the RR of kidney cancer mortality attributable to high BMI increased by 60.80 times and 34.17 times in men and women in China, respectively; in U.S., it increased by 69.68 times 
Table 1 The relative risks of kidney cancer mortality attributable to high BMI due to age, period and cohort effects, China

\begin{tabular}{|c|c|c|c|c|c|c|}
\hline \multirow[t]{3}{*}{ Factor } & \multicolumn{3}{|l|}{ Men } & \multicolumn{3}{|c|}{ Women } \\
\hline & \multirow[t]{2}{*}{$\overline{\mathrm{RR}}$} & \multicolumn{2}{|l|}{$95 \% \mathrm{Cl}$} & \multirow[t]{2}{*}{$\overline{R R}$} & \multicolumn{2}{|l|}{$95 \% \mathrm{Cl}$} \\
\hline & & Lower & Upper & & Lower & Upper \\
\hline \multicolumn{7}{|l|}{ Age } \\
\hline $20-24$ & 0.07 & 0.00 & $4.93 \times 10^{5}$ & 0.11 & 0.00 & $2.52 \times 10^{5}$ \\
\hline $25-29$ & 0.13 & 0.00 & 2322.96 & 0.17 & 0.00 & 3853.25 \\
\hline $30-34$ & 0.21 & 0.00 & 410.40 & 0.20 & 0.00 & 1275.74 \\
\hline $35-39$ & 0.31 & 0.00 & 168.34 & 0.25 & 0.00 & 478.42 \\
\hline $40-44$ & 0.50 & 0.00 & 89.29 & 0.40 & 0.00 & 159.30 \\
\hline $45-49$ & 0.82 & 0.01 & 60.57 & 0.67 & 0.01 & 83.08 \\
\hline $50-54$ & 1.22 & 0.03 & 47.55 & 0.98 & 0.02 & 57.00 \\
\hline $55-59$ & 1.62 & 0.07 & 36.81 & 1.40 & 0.05 & 41.47 \\
\hline $60-64$ & 1.91 & 0.14 & 26.61 & 2.03 & 0.13 & 31.29 \\
\hline $65-69$ & 2.23 & 0.25 & 19.89 & 2.74 & 0.30 & 25.00 \\
\hline $70-74$ & 2.63 & 0.42 & 16.29 & 3.29 & 0.51 & 21.10 \\
\hline $75-79$ & 3.50 & 0.73 & 16.75 & 3.52 & 0.62 & 19.90 \\
\hline $80-84$ & 3.57 & 0.71 & 17.98 & 3.34 & 0.51 & 21.85 \\
\hline $85-89$ & 4.07 & 0.63 & 26.21 & 3.96 & 0.45 & 34.84 \\
\hline $90-94$ & 3.96 & 0.40 & 39.22 & 3.63 & 0.25 & 52.85 \\
\hline \multicolumn{7}{|l|}{ Period } \\
\hline 1992 & 0.39 & 0.05 & 2.85 & 0.49 & 0.06 & 3.99 \\
\hline 1997 & 0.49 & 0.11 & 2.16 & 0.59 & 0.12 & 2.80 \\
\hline 2002 & 0.82 & 0.28 & 2.38 & 0.85 & 0.27 & 2.70 \\
\hline 2007 & 1.31 & 0.50 & 3.40 & 1.18 & 0.40 & 3.44 \\
\hline 2012 & 1.98 & 0.61 & 6.47 & 1.68 & 0.45 & 6.22 \\
\hline 2017 & 2.44 & 0.50 & 11.88 & 2.05 & 0.36 & 11.79 \\
\hline \multicolumn{7}{|l|}{ Cohort } \\
\hline 1902-1906 & 3.29 & 0.04 & 276.04 & 2.70 & 0.02 & 355.62 \\
\hline 1907-1911 & 2.68 & 0.08 & 91.34 & 2.40 & 0.05 & 110.30 \\
\hline 1912-1916 & 2.27 & 0.12 & 41.76 & 2.15 & 0.09 & 50.90 \\
\hline 1917-1921 & 1.97 & 0.17 & 22.35 & 1.90 & 0.13 & 27.13 \\
\hline 1922-1926 & 1.89 & 0.24 & 15.10 & 1.80 & 0.18 & 17.56 \\
\hline 1927-1931 & 1.76 & 0.26 & 11.84 & 1.74 & 0.22 & 13.98 \\
\hline 1932-1936 & 1.55 & 0.20 & 12.23 & 1.64 & 0.17 & 15.64 \\
\hline 1937-1941 & 1.35 & 0.12 & 14.80 & 1.52 & 0.11 & 20.67 \\
\hline 1942-1946 & 1.11 & 0.07 & 18.81 & 1.37 & 0.06 & 28.99 \\
\hline 1947-1951 & 1.01 & 0.04 & 28.23 & 1.27 & 0.04 & 45.26 \\
\hline 1952-1956 & 1.04 & 0.02 & 45.92 & 1.26 & 0.02 & 78.05 \\
\hline 1957-1961 & 0.92 & 0.01 & 69.02 & 1.06 & 0.01 & 127.64 \\
\hline 1962-1966 & 0.78 & 0.01 & 103.57 & 0.82 & 0.00 & 216.10 \\
\hline 1967-1971 & 0.78 & 0.00 & 170.81 & 0.78 & 0.00 & 387.01 \\
\hline 1972-1976 & 0.64 & 0.00 & 295.45 & 0.63 & 0.00 & 787.25 \\
\hline 1977-1981 & 0.50 & 0.00 & 769.12 & 0.48 & 0.00 & 3280.14 \\
\hline 1982-1986 & 0.45 & 0.00 & 2792.20 & 0.42 & 0.00 & $17,949.19$ \\
\hline
\end{tabular}

Table 1 The relative risks of kidney cancer mortality attributable to high BMI due to age, period and cohort effects, China (Continued)

\begin{tabular}{|c|c|c|c|c|c|c|}
\hline \multirow[t]{3}{*}{ Factor } & \multicolumn{3}{|c|}{ Men } & \multicolumn{3}{|c|}{ Women } \\
\hline & \multirow[t]{2}{*}{$\overline{R R}$} & \multicolumn{2}{|l|}{$95 \% \mathrm{Cl}$} & \multirow[t]{2}{*}{$\overline{\mathrm{RR}}$} & \multicolumn{2}{|l|}{$95 \% \mathrm{Cl}$} \\
\hline & & Lower & Upper & & Lower & Upper \\
\hline 1987-1991 & 0.42 & 0.00 & $21,024.40$ & 0.38 & 0.00 & $1.23 \times 10^{5}$ \\
\hline 1992-1996 & 0.37 & 0.00 & $2.15 \times 10^{6}$ & 0.32 & 0.00 & $6.76 \times 10^{6}$ \\
\hline $1997-2001$ & 0.26 & 0.00 & $1.36 \times 10^{14}$ & 0.21 & 0.00 & $4.28 \times 10^{1}$ \\
\hline
\end{tabular}

and 55.23 times in men and women, respectively (see Supplementary Table 2A and 2B).

\section{Period effect}

The period effect on the mortality continuously increased in both China and U.S. adults, while it rapidly increased in China than U.S. during the observation period (Fig. 3b, Tables 1 and 2). This trend indicates that the RR of kidney cancer mortality attributable to high BMI increased with advancing time, and Chinese population is being more exposed to an increased risk of kidney cancer deaths. From 1992 to 2017, the RR of the mortality increased by 6.23 times and 4.14 times in men and women in China, respectively; in U.S., it increased by 2.53 times and 2.05 times in men and women, respectively (see Supplementary Table $2 \mathrm{~A}$ and 2B). Additionally, men have a higher risk than women.

\section{Cohort effect}

The cohort effect continuously decreased in China, and a decreasing trend was also observed in U.S. with exception of 1902-1906 and 1907-1921 birth cohort (Fig. 3c, Tables 1 and 2). From 1902-1906 to 1997-2001 birth cohort, the RR of kidney cancer mortality attributable to high BMI decreased by 92.24 and $92.29 \%$ in men and women in China, respectively; in U.S., the RR of that decreased by 87.54 and $87.64 \%$ in men and women, respectively (see Supplementary Table 2A and 2B).

\section{Discussion}

The causes of kidney cancer are very complex, and the relevant literature shows that smoking, hypertension and obesity are the three most important risk factors [27]. According to relevant studies, the summary relative risk estimate was 1.07 (95\% CI 1.05-1.09) per unit of increase in BMI [28]. With the socio-economic development, urbanization, intensified industrialization and changes in dietary and lifestyles, the exposure to kidney cancer risk factors in Chinese population has increased. From 1980 to 2000, global tobacco consumption showed an upward trend in most regions, and tobacco consumption in China increased the fastest; but decreased by $27 \%$ 
Table 2 The relative risks of kidney cancer mortality attributable to high BMI due to age, period and cohort effects, U.S.

\begin{tabular}{|c|c|c|c|c|c|c|}
\hline \multirow[t]{3}{*}{ Factor } & \multicolumn{3}{|l|}{ Men } & \multicolumn{3}{|c|}{ Women } \\
\hline & \multirow[t]{2}{*}{$\mathrm{RR}$} & \multicolumn{2}{|l|}{$95 \% \mathrm{Cl}$} & \multirow[t]{2}{*}{$\mathrm{RR}$} & \multicolumn{2}{|l|}{$95 \% \mathrm{Cl}$} \\
\hline & & Lower & Upper & & Lower & Upper \\
\hline \multicolumn{7}{|l|}{ Age } \\
\hline $20-24$ & 0.05 & 0.00 & 13.34 & 0.08 & 0.00 & 17.08 \\
\hline $25-29$ & 0.07 & 0.00 & 4.30 & 0.11 & 0.00 & 6.18 \\
\hline $30-34$ & 0.12 & 0.01 & 2.80 & 0.14 & 0.00 & 4.35 \\
\hline $35-39$ & 0.25 & 0.02 & 2.48 & 0.24 & 0.02 & 3.41 \\
\hline $40-44$ & 0.54 & 0.09 & 3.08 & 0.43 & 0.05 & 3.42 \\
\hline $45-49$ & 1.06 & 0.26 & 4.25 & 0.76 & 0.15 & 3.97 \\
\hline $50-54$ & 1.70 & 0.54 & 5.36 & 1.24 & 0.32 & 4.73 \\
\hline $55-59$ & 2.38 & 0.93 & 6.06 & 1.79 & 0.60 & 5.32 \\
\hline $60-64$ & 2.88 & 1.35 & 6.13 & 2.37 & 0.99 & 5.71 \\
\hline $65-69$ & 3.18 & 1.73 & 5.85 & 2.89 & 1.43 & 5.85 \\
\hline $70-74$ & 3.31 & 1.96 & 5.59 & 3.35 & 1.84 & 6.09 \\
\hline $75-79$ & 3.38 & 2.00 & 5.70 & 3.55 & 1.96 & 6.42 \\
\hline $80-84$ & 3.07 & 1.66 & 5.68 & 3.73 & 1.88 & 7.38 \\
\hline 85-89 & 3.49 & 1.65 & 7.38 & 4.13 & 1.79 & 9.54 \\
\hline $90-94$ & 3.50 & 1.38 & 8.84 & 4.18 & 1.48 & 11.77 \\
\hline \multicolumn{7}{|l|}{ Period } \\
\hline 1992 & 0.62 & 0.34 & 1.14 & 0.70 & 0.36 & 1.37 \\
\hline 1997 & 0.76 & 0.51 & 1.14 & 0.82 & 0.52 & 1.29 \\
\hline 2002 & 0.94 & 0.72 & 1.21 & 0.95 & 0.70 & 1.29 \\
\hline 2007 & 1.09 & 0.84 & 1.42 & 1.07 & 0.79 & 1.46 \\
\hline 2012 & 1.30 & 0.88 & 1.93 & 1.21 & 0.77 & 1.90 \\
\hline 2017 & 1.58 & 0.90 & 2.78 & 1.43 & 0.75 & 2.71 \\
\hline \multicolumn{7}{|l|}{ Cohort } \\
\hline 1902-1906 & 2.31 & 0.54 & 9.95 & 2.31 & 0.47 & 11.21 \\
\hline 1907-1911 & 2.45 & 0.76 & 7.91 & 2.44 & 0.69 & 8.65 \\
\hline 1912-1916 & 2.56 & 0.97 & 6.79 & 2.52 & 0.89 & 7.19 \\
\hline 1917-1921 & 2.52 & 1.11 & 5.71 & 2.51 & 1.05 & 6.02 \\
\hline 1922-1926 & 2.42 & 1.19 & 4.95 & 2.39 & 1.12 & 5.10 \\
\hline 1927-1931 & 2.21 & 1.13 & 4.31 & 2.17 & 1.06 & 4.42 \\
\hline 1932-1936 & 1.97 & 0.96 & 4.01 & 1.93 & 0.89 & 4.19 \\
\hline 1937-1941 & 1.72 & 0.76 & 3.90 & 1.70 & 0.69 & 4.21 \\
\hline 1942-1946 & 1.49 & 0.57 & 3.90 & 1.46 & 0.50 & 4.29 \\
\hline 1947-1951 & 1.26 & 0.41 & 3.89 & 1.22 & 0.34 & 4.40 \\
\hline 1952-1956 & 1.03 & 0.28 & 3.85 & 1.00 & 0.22 & 4.53 \\
\hline 1957-1961 & 0.86 & 0.19 & 3.93 & 0.85 & 0.15 & 4.90 \\
\hline 1962-1966 & 0.70 & 0.12 & 4.02 & 0.72 & 0.10 & 5.40 \\
\hline 1967-1971 & 0.56 & 0.08 & 4.17 & 0.57 & 0.06 & 5.99 \\
\hline 1972-1976 & 0.46 & 0.04 & 4.74 & 0.46 & 0.03 & 7.37 \\
\hline 1977-1981 & 0.40 & 0.02 & 7.14 & 0.41 & 0.01 & 12.41 \\
\hline 1982-1986 & 0.38 & 0.01 & 15.64 & 0.40 & 0.01 & 26.98 \\
\hline
\end{tabular}

Table 2 The relative risks of kidney cancer mortality attributable to high BMI due to age, period and cohort effects, U.S.

(Continued)

\begin{tabular}{|c|c|c|c|c|c|c|}
\hline \multirow[t]{3}{*}{ Factor } & \multicolumn{3}{|c|}{ Men } & \multicolumn{3}{|c|}{ Women } \\
\hline & \multirow[t]{2}{*}{$\overline{\mathrm{RR}}$} & \multicolumn{2}{|l|}{$95 \% \mathrm{Cl}$} & \multirow[t]{2}{*}{$\overline{\mathrm{RR}}$} & \multicolumn{2}{|l|}{$95 \% \mathrm{Cl}$} \\
\hline & & Lower & Upper & & Lower & Upper \\
\hline 1987-1991 & 0.36 & 0.00 & 52.63 & 0.37 & 0.00 & 83.92 \\
\hline 1992-1996 & 0.33 & 0.00 & 363.70 & 0.33 & 0.00 & 483.03 \\
\hline $1997-2001$ & 0.29 & 0.00 & $90,958.62$ & 0.28 & 0.00 & $1.33 \times 10^{5}$ \\
\hline
\end{tabular}

Notes: $R R$ Relative risk [RR $=$ exp. $($ coefficient)], $C /$ Confidence interval

and $2 \%$ in the Americas and Europe [29]. According to the Chinese national nutrition and health survey, the prevalence of hypertension in the population aged over 15 years old was $5.1 \%$ in $1958-1959,7.7 \%$ in $1979-1980$, $13.6 \%$ in 1991, and $17.6 \%$ in 2002 , and the agestandardized rate also showed the upward trend [15]. In 1982, overweight and obesity among the Chinese population were still very rare, at $6 \%$ and $0.6 \%$, respectively. In 2014, the overweight rate reached $34.26 \%$ and the obesity rate was $10.98 \%$ [15]. In overall, all these factors may be related to the increased mortality of kidney cancer in this present study.

Obesity/overweight is considered to be the increased risk for kidney cancer $[8,14]$. The prevalence of obesity/ overweight is different between China and the U.S. Our study found that kidney cancer deaths attributable to high BMI showed an increasing tendency in China and men U.S., and the overall mortality rate of kidney cancer is still increasing in both genders in China but not in the U.S. Comparison to U.S., China has a serious attribution burden of kidney cancer death and BMI. The exposure to kidney cancer risk factors in Chinese population is increasing yearly. Potential kidney cancer risk factors include behavioural and environmental factors, comorbidities, and analgesics. Smoking, obesity, and hypertension represent established risk factors [30]. In GBD 2017 study, spatiotemporal Gaussian process regression was used to estimate risk-attributable burden and risk exposure for many risks, typically those with rich age-sex-specific data. It synthesises noisy data by borrowing strength across space, time, and age to best estimate the underlying trends for a given risk. GBD study found considerable heterogeneity across superregions in the leading risk factors. There are also marked spatial patterns for other risks such as high BMI in central America, north Africa and the Middle East, and Oceania [22]. In our study, we obtained China and U.S. mortality data from GBD study to analyze the relationships between kidney cancer deaths and high BMI. The geographic heterogeneity in mortality rates of kidney cancer attributable to high BMI may be considerable between China and U.S. 


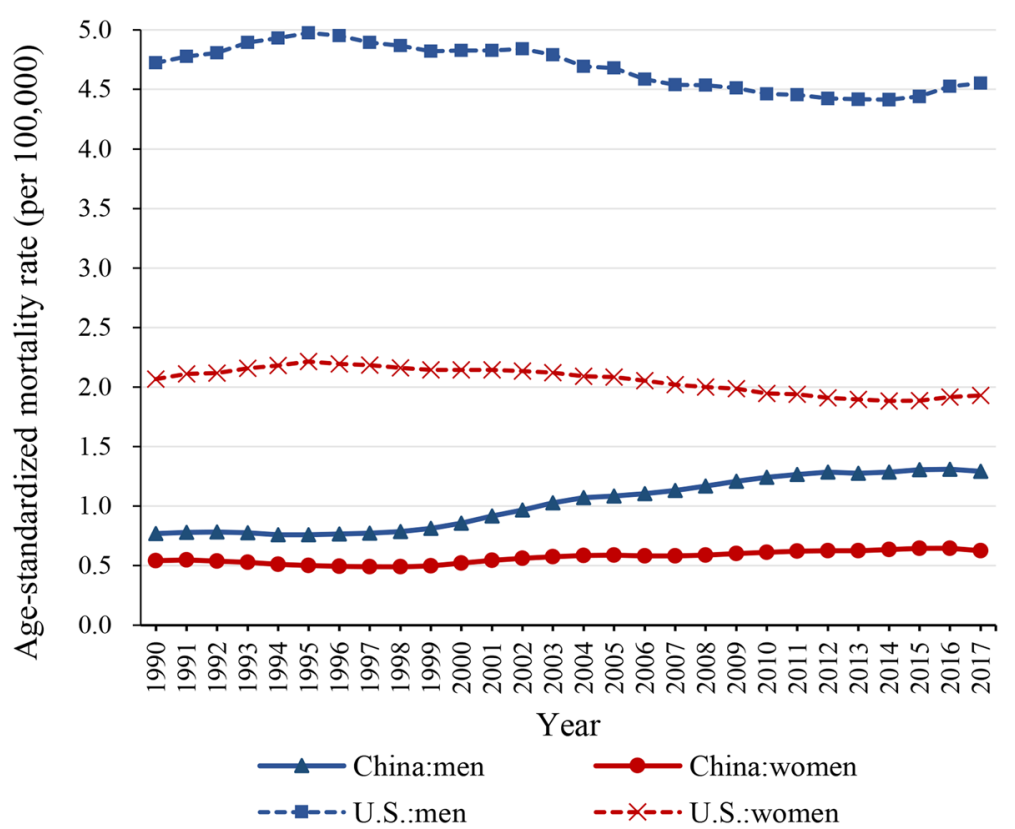

Fig. 1 The age-standardized mortality rates of kidney cancer in China and the U.S. for both sexes at all ages, 1990-2017. Blue corresponds to men. Red corresponds to women

In China, the increasing ASMR of kidney cancer attributable to high BMI was possibly associated with the prevalence of obesity $[12,13]$. The increasing attributable burden and death of kidney cancer may be related to the improved diagnosis level of kidney cancer [6], and changes in exposure levels of kidney cancer risk factors in the population also have impacts on the mortality of kidney cancer [31]. Differently, a stable trend or slight decline was observed for kidney cancer mortality in U.S., which may be explained by effective improving the rising obesity trend in the US adult population over the past decades [32, 33]. In U.S., relevant researches reported that obesity prevalence remains need continue surveillance [34], and the increasing mortality attributable to

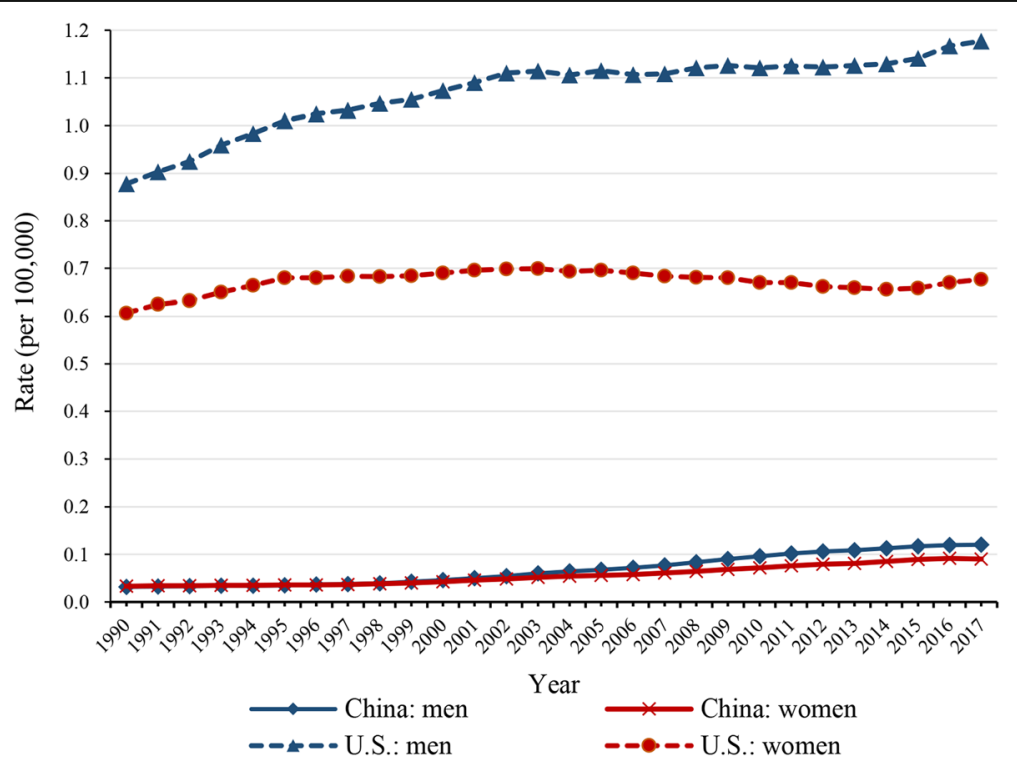

Fig. 2 Trends in the age-standardized rates of kidney cancer mortality attributable to high BMI in China and the U.S. from 1990 to 2017 , at all ages. Blue corresponds to men. Red corresponds to women 

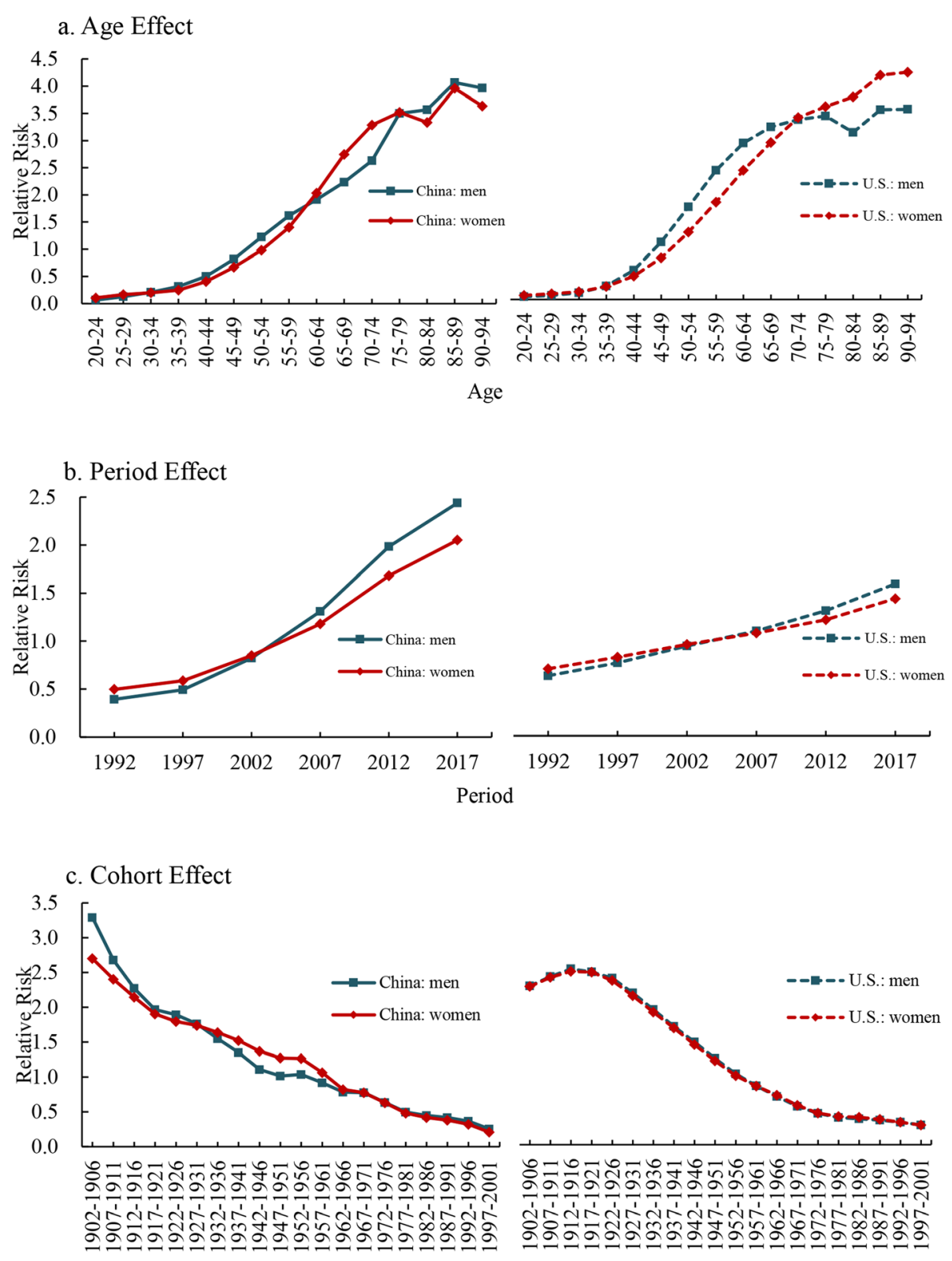

Cohort

Fig. 3 Kidney cancer mortality attributable to high BMI relative risks due to (a) age; (b) period; and (c) cohort effects. Blue corresponds to men. Red corresponds to women

high BMI was observed in men U.S. Thus, this attributable burden about BMI and kidney cancer remains need be focused.

After adjusting the three effects using the APC model, we confirmed that the differences in age-specific mortality rate patterns exist. A similar trend of age effect on kidney cancer mortality attributable to high BMI was observed between China and the U.S. adults. The age effect increased in the two areas, which indicated that the risk of the mortality of kidney cancer attributable to high BMI tended to increase in the middle-aged and younger groups. This finding may suggest that aging has driven the trend of the mortality $[35,36]$. The interesting phenomenon is that age effect in the two areas was found to increase exponentially until age 70 , when it continues to rise, albeit at a slower pace.

The cohort effects of the youngest and oldest age groups must be interpreted carefully because they are the small number of observations and they have larger standard errors than estimates for the middle cohorts [37]. Overall, The general trends of cohort effect showed a continuously declined trend, which indicated a 
decreased risk of the mortality in younger generations. Younger generations may receive good education and have a strong awareness of health [38], which may have a connection with the mortality of kidney cancer. For example, earlier birth cohorts had weak health awareness and they realized neither the occurrence of cancer nor the damage of obesity or overweight to human health. It is worth noting that cohort effect increased for 19021906 and 1907-1911 birth cohort in the U.S., which may indicate a higher risk in the earliest birth cohort. This finding should be treated carefully.

Here, period effects were found to be small or modest when birth cohort and age effects were both controlled. This study showed that the period effect might be the critical factor in the trend of kidney cancer mortality, because the period effect in China was significantly continuously increasing and its mortality rate was also continuously, while the period effect in U.S. slightly increased over the same period and its mortality rate tends to be stable. This relationship needs to be further verified. Increasing period effect was observed in both China and U.S. adults over the whole study period, which indicated the period risk factors might be contributed to the increase in the linear trend of the age-standardized mortality rate of kidney cancer attributable to high BMI through 1990 to 2017. However, the period trend increased rapidly in Chinese adults, compared with U.S. adults. The difference in period effect of kidney cancer was possibly related to the different risk exposure. In U.S., no significant changes in obesity prevalence in recent years [34], and some measures were conducted for improving the increasing obesity prevalence in U.S. adults [32, 33]. However, the prevalence of childhood obesity in the U.S. is rising during the past decades [39], and an increasing trend in obesity was observed for U.S. women during 2005-2014 [40]. Thus, this finding also needs to be further verified. Traditionally, obesity has been considered a problem in Western countries, while urbanisation in Asia has led to a sedentary lifestyle and overnutrition, setting the stage for the epidemic of obesity [41]. According to the 2011 China Health and Nutrition Survey, the prevalence of obesity among both Chinese adults increased significantly over the past decades, especially in men [42, 43]. Moreover, Chinese children may have a severe obesity problem. National study reported that $14.4 \%$ of children and adolescents were overweight, $11.9 \%$ were obese, and $36.8 \%$ did not meet screen-time viewing recommendations [44, 45]. The possible reason of difference between the two areas was associated to the increasing obesity prevalence. As for the different increasing period effect, the underlying reason of different extent to the trend between the two areas was possibly attributable to the fact that the increase in adult obesity in U.S. has slowed down [46].
Thus, Chinese population seems to face a more severe situation of attributable burden of kidney cancer. Our findings also may indicate inadequate measures or policies on obesity prevalence in Chinese adults, while U.S. has efforts in prevention and care of obesity, and in establishing collaborative weight management models [47, 48], and the rates of awareness, treatment and control of disease are relatively low in China population [5]. In addition, there have a higher relative risk of the mortality of kidney cancer attributable to high BMI in men than women. In China, it is more likely to be overweight/obesity in men compared with women [42, 49]. This gender difference in the relationship of kidney cancer and obesity should be further studied.

In summary, there has rapid urbanization and increasing prevalence of obesity in China, and previous study also noted a decrease in all measures of physical fitness in normal-weight adults during 2000-2014 [13], the risk of kidney cancer and high BMI need be focused. The increasing period effect indicated the period factors may be the key factor affecting the increasing mortality of kidney cancer. Thus effective measures such as promoting national fitness and low-fat dietary are needed for the prevention of obesity/overweight. It is necessary to reduce kidney cancer deaths in Chinese adults.

\section{Limitations}

There are also some limitations in the present study. First, the data from the GBD study were supplied by the governments of the various countries and districts, which may have substantially different systems for collecting vital statistics and methods used to confirm causes of death. These factors limit the comparability of the information in the two areas. Second, despite the mortality data estimated by GBD study which incorporates methods to adjust for incomplete or missing VR and VA data, general heterogeneity in data completeness and quality, and the redistribution of so-called garbage codes (insufficiently specific or implausible cause of death codes), there might be difficult to thoroughly avoid inaccuracy of data. Therefore, our results in the present study on epidemiology of kidney cancer mortality should be treated carefully.

\section{Conclusions}

The age-standardized morality rate of kidney cancer attributable to high BMI is rapidly increasing in China, while the mortality rate in women U.S. varies and tend to be stable in recent years. Apart from that, age effect increased and cohort effect decreased in both China and the U.S. adults, while period effect increased rapidly in China adults, compared with the U.S. adults. Obesity prevalence and China's aging also may continuously drive kidney cancer death. Effective mearsures, such as 
the correct knowledge and adopting policies on body weight control and care, should be noted and conducted.

\section{Supplementary information}

Supplementary information accompanies this paper at https://doi.org/10. 1186/s12889-020-09007-7.

Additional file 1: Supplementary Table 1. Goodness-of-fit in APC models for mortality of kidney cancer attributable to high BMI. Supplementary Table 2A. Kidney cancer mortality attributable to high BMI estimated coefficients for the age, period and cohort effects, men.

Supplementary Table 2B. Kidney cancer mortality attributable to high BMI estimated coefficients for the age, period and cohort effects, women.

\section{Abbreviations}

BMI: Body-mass index; APC: Age, period, and cohort; RR: Relative risk; ASMR: Age-standardized mortality rates; GBD: Global burden of disease; CDC: Center for Disease Control and Prevention; DSPs: Disease Surveillance Points; CODEm: Cause of Death Ensemble model; VR: Vital registration; VA: Verbal autopsy; ICD: International Classification of Diseases; CGLIMs: Constrained generalized linear models

\section{Acknowledgements}

We appreciate the works by the Global Burden of Disease study2017 collaborators.

\section{Authors' contributions}

$X L$ and $C Y$ performed the study design, data collection, and wrote the manuscript. $X L$ completed first draft of the manuscript. $C Y$ completed final version of the manuscript and supervised all of the work. CY, YY, MW, FW, SM, YW, and RM checked and revised the manuscript. All authors read and approved the final manuscript.

\section{Funding}

The study is part of the first author's PhD research. The PhD research was funded by the National Key Research and Development Program of China [grant numbers 2018YFC1315302, 2017YFC1200502] and the National Natural Science Foundation of China [grant number 81773552].

\section{Availability of data and materials}

The datasets generated and/or analysed during the current study are available in the GBD repository, which was publically available at http://ghdx. healthdata.org/gbd-results-tool.

\section{Ethics approval and consent to participate} Not applicable.

\section{Consent for publication}

Not applicable.

\section{Competing interests}

The authors declare that they have no competing interests.

\section{Author details \\ 'Department of Epidemiology and Biostatistics, School of Health Sciences, Wuhan University, No. 115, Donghu Road, Wuhan 430071, China. ${ }^{2}$ School of Public Health and Management, Hubei University of Medicine, Shiyan 442000, China. ${ }^{3}$ The First Affiliated Hospital of Anhui Medical University, Anhui 230022, China. ${ }^{4}$ Global Health Institute, Wuhan University, Wuhan 430072, China.}

Received: 2 March 2020 Accepted: 27 May 2020

Published online: 08 June 2020

\section{References}

1. Owens B. Kidney cancer. Nature. 2016;537(7620):S97.

2. Disease GBD, Injury I, Prevalence C. Global, regional, and national incidence, prevalence, and years lived with disability for 354 diseases and injuries for
195 countries and territories, 1990-2017: a systematic analysis for the global burden of Disease study 2017. Lancet. 2018:392(10159):1789-858.

3. Ljungberg B, Campbell SC, Choi HY, Jacqmin D, Lee JE, Weikert S, et al. The epidemiology of renal cell carcinoma. Eur Urol. 2011;60(4):615-21.

4. Siegel RL, Miller KD, Jemal A. Cancer statistics, 2015. CA Cancer J Clin. 2015; 65(1):5-29.

5. Scelo G, Larose TL. Epidemiology and risk factors for kidney cancer. J Clin Oncol. 2018; 36(36):JCO2018791905.

6. Liu SZ, Guo LW, Cao XQ, Chen Q, Zhang SK, Zhang M, et al. Estimation on the incidence and mortality of kidney cancer in China, in 2014. Zhonghua Liu Xing Bing Xue Za Zhi. 2018;39(10):1346-50.

7. Hidayat K, Du X, Zou SY, Shi BM. Blood pressure and kidney cancer risk: meta-analysis of prospective studies. J Hypertens. 2017;35(7):1333-44.

8. Liu XZ, Sun Q, Hou HF, Zhu K, Wang Q, Liu HM, et al. The association between $\mathrm{BMI}$ and kidney cancer risk An updated dose-response meta-analysis in accordance with PRISMA guideline. Medicine. 2018;97(44):e1 2860.

9. Gati A, Kouidhi S, Marrakchi R, El Gaaied A, Kourda N, Derouiche A, et al. Obesity and renal cancer: role of adipokines in the tumor-immune system conflict. Oncoimmunology. 2014;3(1):e27810.

10. Solarek W, Czarnecka AM, Escudier B, Bielecka ZF, Lian F, Szczylik C. Insulin and IGFs in renal cancer risk and progression. Endocr Relat Cancer. 2015; 22(5):R253-64.

11. Tuomi T, Santoro N, Caprio S, Cai M, Weng J, Groop L. The many faces of diabetes: a disease with increasing heterogeneity. Lancet. 2014;383(9922): 1084-94.

12. Xi B, Liang Y, He T, Reilly KH, Hu Y, Wang $Q$, et al. Secular trends in the prevalence of general and abdominal obesity among Chinese adults, 19932009. Obes Rev. 2012;13(3):287-96.

13. Tian $Y$, Jiang $C M$, Wang $M$, Cai $R$, Zhang $Y F$, He ZH, et al. BMI, leisure-time physical activity, and physical fitness in adults in China: results from a series of national surveys, 2000-14. Lancet Diabetes Endocrinol. 2016;4(6):487-97.

14. Wang F, Xu Y. Body mass index and risk of renal cell cancer: a doseresponse meta-analysis of published cohort studies. Int J Cancer. 2014; 135(7):1673-86.

15. Li LM, Rao KQ, Kong LZ, Yao CH, Xiang HD, Zhai FY, et al. A description on the Chinese national nutrition and health survey in 2002. Zhonghua Liu Xing Bing Xue Za Zhi. 2005;26(7):478-84.

16. Collaborators GBDCoD. Global, regional, and national age-sex-specific mortality for 282 causes of death in 195 countries and territories, 1980-2017: a systematic analysis for the global burden of Disease study 2017. Lancet. 2018;392(10159):1736-88.

17. Mortality GBD. Causes of death C. global, regional, and national life expectancy, all-cause mortality, and cause-specific mortality for 249 causes of death, 1980-2015: a systematic analysis for the global burden of Disease study 2015. Lancet. 2016;388(10053):1459-544.

18. Murray CJ, Lopez AD, Jamison DT. The global burden of disease in 1990: summary results, sensitivity analysis and future directions. Bull World Health Organ. 1994;72(3):495-509.

19. Global Burden of Disease Cancer C, Fitzmaurice C, Akinyemiju TF, Al Lami FH, Alam T, Alizadeh-Navaei R, et al. Global, regional, and national incidence, prevalence, and years lived with disability for 354 diseases and injuries for 195 countries and territories, 1990-2017: a systematic analysis for the Global Burden of Disease Study 2017. Lancet. 2018:392:1789-858.

20. Collaboration GBDCKD. Global, regional, and national burden of chronic kidney disease, 1990-2017: a systematic analysis for the global burden of Disease study 2017. Lancet. 2020;395(10225):709-33.

21. Zhou M, Wang H, Zhu J, Chen W, Wang L, Liu S, et al. Cause-specific mortality for 240 causes in China during 1990-2013: a systematic subnational analysis for the global burden of Disease study 2013. Lancet. 2016;387(10015):251-72.

22. Collaborators GBDRF. Global, regional, and national comparative risk assessment of 84 behavioural, environmental and occupational, and metabolic risks or clusters of risks for 195 countries and territories, 19902017: a systematic analysis for the global burden of Disease study 2017. Lancet. 2018;392(10159):1923-94.

23. Lin L, Yan L, Liu Y, Yuan F, Li H, Ni J. Incidence and death in 29 cancer groups in 2017 and trend analysis from 1990 to 2017 from the global burden of Disease study. J Hematol Oncol. 2019;12(1):96.

24. Wang L, Yu C, Liu Y, Wang J, Li C, Wang Q, et al. Lung Cancer Mortality Trends in China from 1988 to 2013: New challenges and opportunities for the government. Int J Environ Res Public Health. 2016;13(11):1052. 
25. Yang Y, Schulhofer-Wohl S, Fu WJJ, Land KC. The intrinsic estimator for age-periodcohort analysis: what it is and how to use it. Am J Sociol. 2008;113(6):1697-736.

26. Luo G, Zhang Y, Guo P, Wang L, Huang Y, Li K. Global patterns and trends in stomach cancer incidence: age, period and birth cohort analysis. Int J Cancer. 2017;141(7):1333-44.

27. Kabaria R, Klaassen Z, Terris MK. Renal cell carcinoma: links and risks. Int J Nephrol Renov Dis. 2016;9:45-52.

28. Bergstrom A, Hsieh CC, Lindblad P, Lu CM, Cook NR, Wolk A. Obesity and renal cell cancer--a quantitative review. Br J Cancer. 2001;85(7):984-90.

29. Yang GH, Ma JM, Liu N, Zhou LN. Smoking and passive smoking in Chinese, 2002. Zhonghua Liu Xing Bing Xue Za Zhi. 2005;26(2):77-83.

30. Capitanio U, Bensalah K, Bex A, Boorjian SA, Bray F, Coleman J, et al. Epidemiology of renal cell carcinoma. Eur Urol. 2019;75(1):74-84.

31. De P, Otterstatter MC, Semenciw R, Ellison LF, Marrett LD, Dryer D. Trends in incidence, mortality, and survival for kidney cancer in Canada, 1986-2007. Cancer Causes Control. 2014;25(10):1271-81.

32. Chen HJ, Xue H, Liu S, Huang TTK, Wang YC, Wang Y. Obesity trend in the United States and economic intervention options to change it: a simulation study linking ecological epidemiology and system dynamics modeling. Public Health. 2018;161:20-8.

33. LeBlanc ES, Patnode CD, Webber EM, Redmond N, Rushkin M, O'Connor EA. Behavioral and pharmacotherapy weight loss interventions to prevent obesity-related morbidity and Mortality in adults: updated evidence report and systematic review for the US preventive services task force. JAMA. 2018 320(11):1172-91.

34. Ogden CL, Carroll MD, Kit BK, Flegal KM. Prevalence of childhood and adult obesity in the United States, 2011-2012. JAMA. 2014;311(8):806-14.

35. Kanasi E, Ayilavarapu S, Jones J. The aging population: demographics and the biology of aging. Periodontol. 2016;72(1):13-8.

36. Lutz W, Sanderson W, Scherbov S. The coming acceleration of global population ageing. Nature. 2008:451(7179):716-9.

37. Luo L. Assessing validity and application scope of the intrinsic estimator approach to the age-period-cohort problem. Demography. 2013;50(6):1945-67.

38. Cohen AK, Syme SL. Education: a missed opportunity for public health intervention. Am J Public Health. 2013;103(6):997-1001.

39. Skinner AC, Skelton JA. Prevalence and trends in obesity and severe obesity among children in the United States, 1999-2012. JAMA Pediatr. 2014;168(6):561-6.

40. Flegal KM, Kruszon-Moran D, Carroll MD, Fryar CD, Ogden CL. Trends in obesity among adults in the United States, 2005 to 2014. Jama-J Am Med Assoc. 2016;315(21):2284-91.

41. Fan JG, Kim SU, Wong WW. New trends on obesity and NAFLD in Asia. J Hepatol. 2017;67(4):862-73.

42. Mi YJ, Zhang B, Wang HJ, Yan J, Han W, Zhao J, et al. Prevalence and secular trends in obesity among Chinese adults, 1991-2011. Am J Prev Med. 2015;49(5):661-9.

43. Song Y, Ma J, Wang HJ, Wang Z, Hu P, Zhang B, et al. Secular trends of obesity prevalence in Chinese children from 1985 to 2010: urban-rural disparity, Obesity, 2015:23(2):448-53.

44. Cui Z, Huxley R, Wu Y, Dibley MJ. Temporal trends in overweight and obesity of children and adolescents from nine provinces in China from 1991-2006. Int J Pediatr Obes. 2010;5(5):365-74.

45. Cai Y, Zhu X, Wu X. Overweight, obesity, and screen-time viewing among Chinese school-aged children: national prevalence estimates from the 2016 physical activity and fitness in China-the youth study. J Sport Health Sci. 2017:6(4):404-9.

46. Ng M, Fleming T, Robinson M, Thomson B, Graetz N, Margono C, et al. Global, regional, and national prevalence of overweight and obesity in children and adults during 1980-2013: a systematic analysis for the global burden of Disease study 2013. Lancet. 2014:384(9945):766-81.

47. Anderson $\mathrm{KL}$. A review of the prevention and medical management of childhood obesity. Child Adolesc Psychiatr Clin N Am. 2018:27(1):63-76.

48. Bleich SN, Bandara S, Bennett WL, Cooper LA, Gudzune KA. U.S. health professionals' views on obesity care, training, and self-efficacy. Am J Prev Med. 2015:48(4):411-8.

49. Guo $Y$, Yin $X$, Wu H, Chai $X$, Yang $X$. Trends in Overweight and Obesity Among Children and Adolescents in China from 1991 to 2015: A MetaAnalysis. Int J Environ Res Public Health. 2019;16(23):4656.

\section{Publisher's Note}

Springer Nature remains neutral with regard to jurisdictional claims in published maps and institutional affiliations.

Ready to submit your research? Choose BMC and benefit from:

- fast, convenient online submission

- thorough peer review by experienced researchers in your field

- rapid publication on acceptance

- support for research data, including large and complex data types

- gold Open Access which fosters wider collaboration and increased citations

- maximum visibility for your research: over $100 \mathrm{M}$ website views per year

At $\mathrm{BMC}$, research is always in progress.

Learn more biomedcentral.com/submissions 